\title{
THE VEHICLES TRAFFIC FLOW OPTIMIZATION IN AN URBAN TRANSPORTATION SYSTEM BY USING SIMULATION MODELING
}

\author{
Marin-Marian COMAN \\ "Nicolae Bălcescu" Land Forces Academy, Sibiu, Romania \\ com.mary@yahoo.com \\ Dorel BADEA \\ "Nicolae Bălcescu" Land Forces Academy, Sibiu, Romania \\ dorel.badea@yahoo.com
}

\begin{abstract}
The urban transportation system is characterized by the urban roads development and growth of road traffic, which leads, most of the time, to a series of congestions in the vehicles traffic. Consequently, due to a high time duration spent on the road traffic, the travel time from a location to another one could be very upsetting for any car drivers, or embarrassing for emergency services and vehicle convoys that carry goods or sensitive items. Those are mainly reasons for using simulation modeling to analyze and optimize the travel time of the road traffic actors in the an urban transportation system. This paper focuses on optimization vehicles flow in a crowded area of Sibiu city, by using agent-based modeling concept and AnyLogic simulation modeling software.
\end{abstract}

KEYwORDS: simulation modeling, optimization, urban transportation, satellite image, agent-based modeling

\section{Introduction}

The urban transportation infrastructure has a major role in the evolution and development of any city. Well functioning of the urban traffic flow brings a positive impact on population living in the crowded areas from the city.

"For decades, urban transportation policy and practitioners favored a model of analysis that prioritized «mobility» - that is, increasing the speed for moving vehicles, and the time that is saved as a result. While this may make sense on an intuitive level, it is a problematic measure today" (Puentes, 2015).
Usually, when we think about analyzing urban transportation, is taking into consideration lots of factors and appropriate transportation infrastructure such as streets, highways, bike lanes, and public transport lines, etc.

"The ability to realistically predict the demand of transport and traffic on the road network is of critical importance to efficient urban transport and infrastructure planning. Agent based models of urban planning have been increasingly introduced over the last decades. Many agent-based models for transport and urban planning can be found 
in the literature with different geographical scales and at various levels of complexity of agent's behaviors and autonomy. They proved that with a large real world scenario, agent-based modeling, while being able to reproduce the complexity of an urban area and predict emergent behaviors in the area, has no issue with the performance. They also show that for traffic and transport simulation purposes, agent based modeling has been considered as a reliable and well worth developing tool that planners can employ to build and evaluate alternative scenarios of an urban area" (Huynh, Cao, Wickramasuriya, Berryman, Perez \& Barthelemy, 2015).

The modeling process of any system for the purpose to be analyzed and optimized through any type of simulation is hard to be done with conventional methods due to complexity of the actual world and the interdependencies among systems.

The most used and exploited simulations modeling are System Dynamics (SD) and Discrete Events (DE). When a complex system is simulated and analyzed as a whole, the SD approach is more appropriate for that because is linked to feedback loops. Moreover putting in practice the SD approach as a continuous simulation will bring a high abstraction stage of solving problems and therefore is suitable for strategic level. In contrast, the discrete event simulation offers the modeler a possibility to operate with system's models as a discrete sequence of events in time, meaning to have the entities waiting in queues. The Agent-Based Modeling $(\mathrm{ABM})$ is another approach related to simulation modeling, which was recently developed. It is used for modeling the individual entities operating in the system, each of the individual having a distinct behavior and the capacity to learn and adapt to the environment. The entities are called agents and based on the information received they can interact each other manifesting an autonomous behavior.
The modeler will use one of the above-mentioned simulations modeling type based on his experience in the simulation field, the type of system or process, which is to be modeled, and the purpose for using a kind of simulation modeling.

\section{Case Study}

The case study related to vehicles traffic flow optimization is based on urban transportation system of Sibiu city. For tailoring the model that is going to be used for simulation, a satellite image was chosen from the internet, as an open source, with an area of Sibiu city illustrated in the Google map.

Sibiu is a "city located in Transylvania, Romania, with a population of 147,245 . Located some $215 \mathrm{~km}$ (134 mi) north-west of Bucharest, the city straddles the Cibin River, a tributary of the river Olt. Now the capital of Sibiu County, between 1692 and 1791 and 1849-1865 Sibiu was the capital of the Principality of Transylvania. Sibiu is one of the most important cultural centers of Romania and was designated the European Capital of Culture for the year 2007, along with the city of Luxembourg. Formerly the centre of the Transylvanian Saxons, the old city of Sibiu was ranked as "Europe's $8^{\text {th }}-$ most idyllic place to live" by Forbes in 2008" (Primaria Sibiu, 2017).

For this paper, it was been taken into consideration a scenario based on a crowded urban area, a main urban road that crosses the Cibin River and connects the downtown with the city exit. Because city exit passes by the Sibiu International Airport, sometimes, the traffic congestion happens, mainly in the rush hours, resulting in the alteration of the urban mobility transportation system due to vehicle traffic growth and thus a higher number of traffic participants.

The case study scenario is based on following elements: it is considered that 
five vehicles traveling organized as convoy will enter in the city from the southern part of Sibiu in order to load goods and sensitive items from Sibiu Customs located near Sibiu International Airport; then, the convoy will heading to the west to Sibiu exit road. Because of Cibin River, the convoy has to pass the only available road to Sibiu Customs, travelling on Cibin Bridge, and then it will head to Sibiu exit. This route is quite crowded, especially in the beginning and at the end of the week. The problem is how to optimize the vehicles traffic flow in the appointed Sibiu area in order to reduce the duration of convoy traveling from one point to another, taking in consideration the traffic congestion that may appear (Figure no. 1).

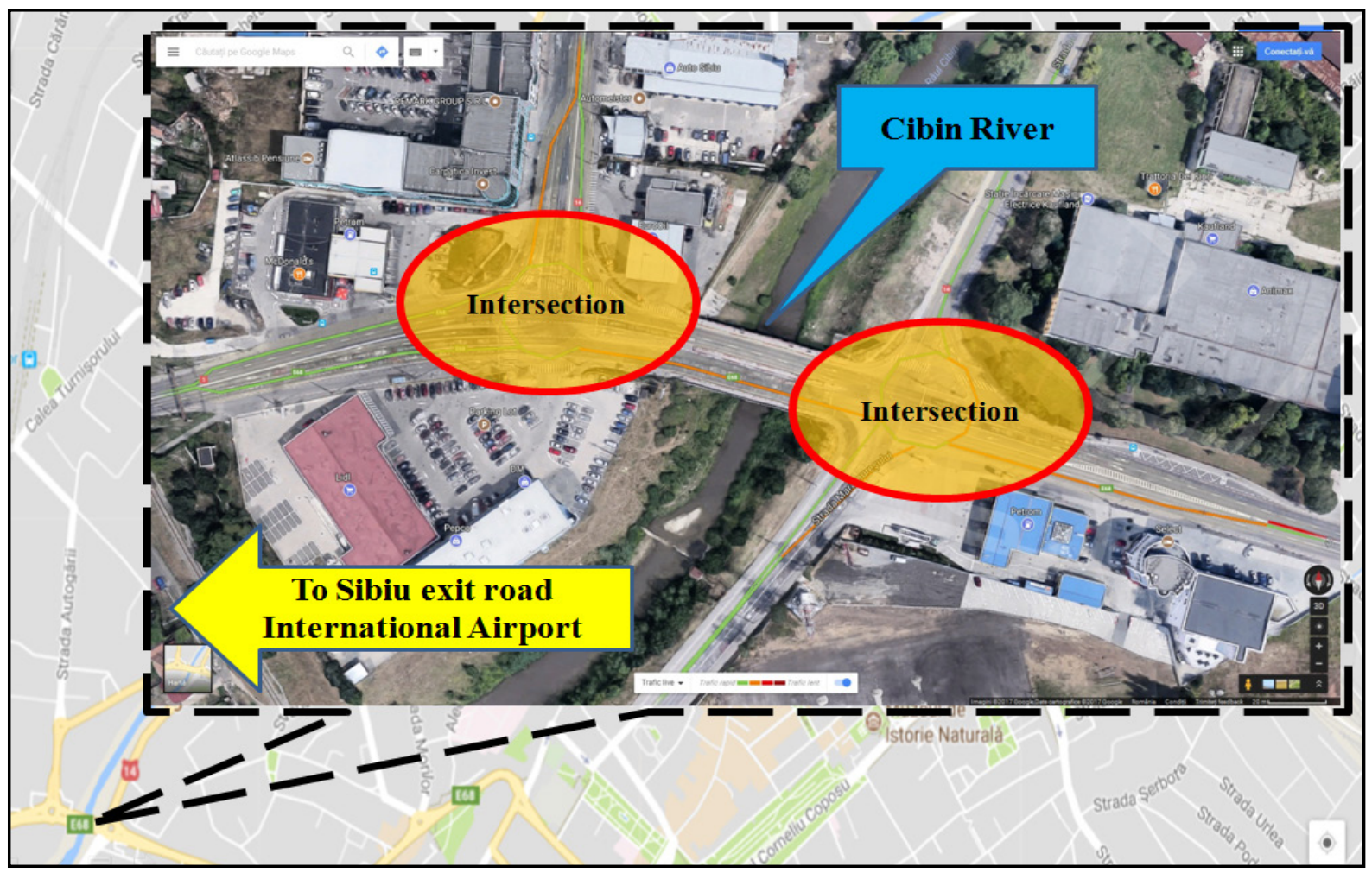

Figure no. 1 Sibiu city-crowded urban area (https://www.google.ro/maps)

In order to solve the optimization problem pertaining to the tailored scenario, it was used the AnyLogic University Researcher v.7.3.6 software to build the simulation model. For doing that, a series of necessary steps were developed.

Step 1. Road network creation

Firstly, a new simulation model was created with the name "Transportation Sibiu city". Then, the satellite image from Google map related to the specified urban area of Sibiu, chosen for the analysis and optimization, was added in the AnyLogic. For good results, the created model had to be scaled with real measurements based on satellite image scale aligned with the AnyLogic scale (in our case the scale was 20 meters).

Secondly, the roads were built and the intersections were created. The intersections were tailored as in reality by taking into considerations the number of the road lanes connections and turning points (Figure no. 2). 


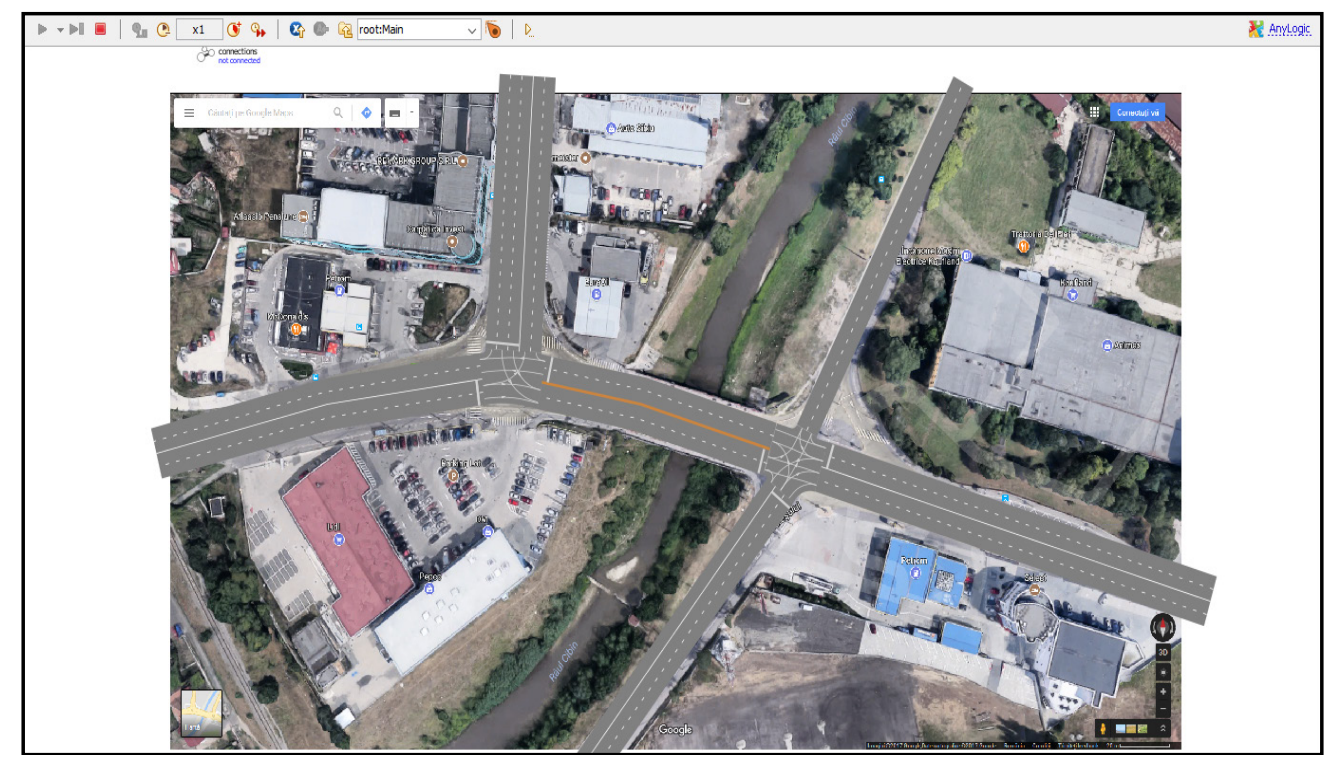

Figure no. 2 Sibiu specified urban area - roads built in AnyLogic

\section{Step 2. Traffic flows logic}

Based on the roads created in the previous step, it was built the flows chart taking into consideration the orientation/ flow of each road lane (forward/backward ways of vehicle traffic).

The flows chart comprises, basically, following elements: carSource, carMoveTo, carDispose, and their logic connections (selectOutput). The selectOutput element has two or five connectors, in order to provide the possibility for each intersection to be tailored as in reality, based on how many traveling directions could be used by the vehicles in the traffic flow. If a car has the possibility to turn right, go straight ahead, and turn left, then it is suitable for using the selectOutput5 with more connectors; also, a probability to use a specific road lane was setup for each of it based on the cars options to follow a particular route.

Then, we paired each segment of the road with the specific elements (Figure no. 3)

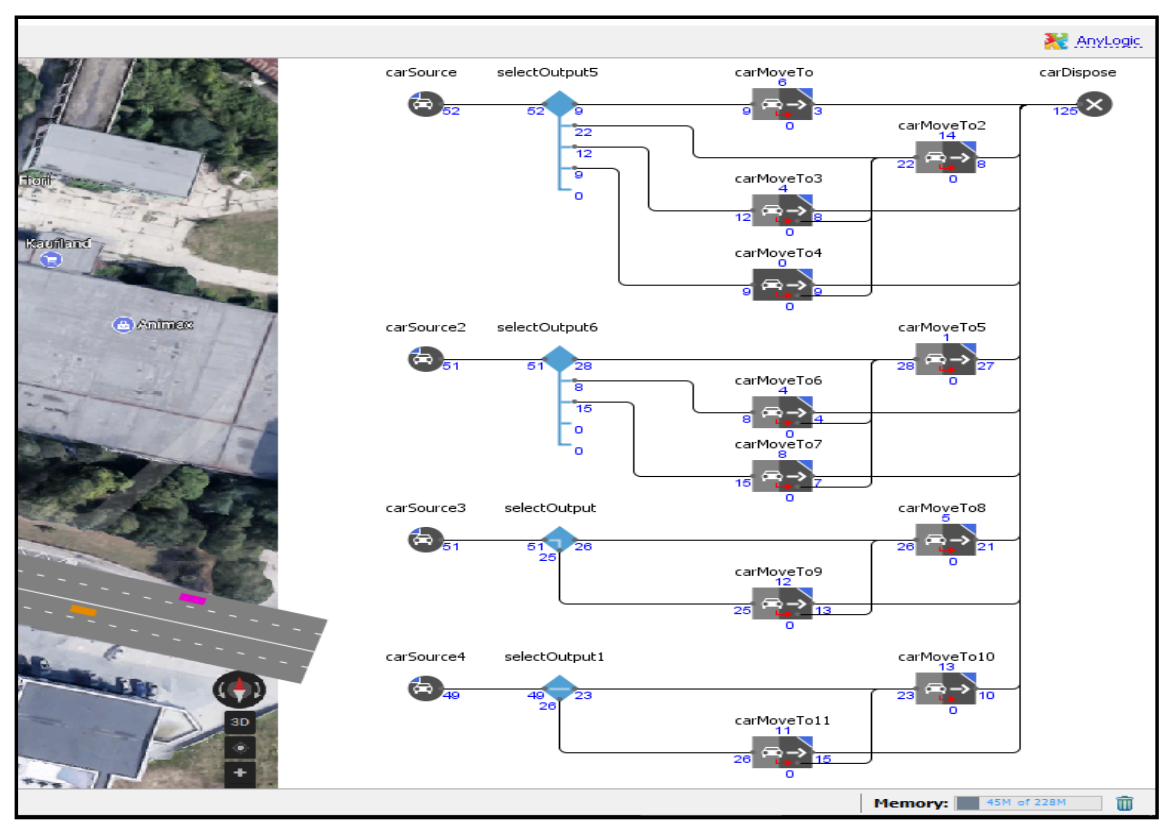

Figure no. 3 The flows chart of the urban transportation model 
Step 3. Traffic lights setup

After flows chart building, the model has been simulated in order to notice any improper functioning.
For intersections is mandatory to have set up the traffic lights (Figure no. 4, 5). If not, the AnyLogic will display a malfunction warning in our simulation.

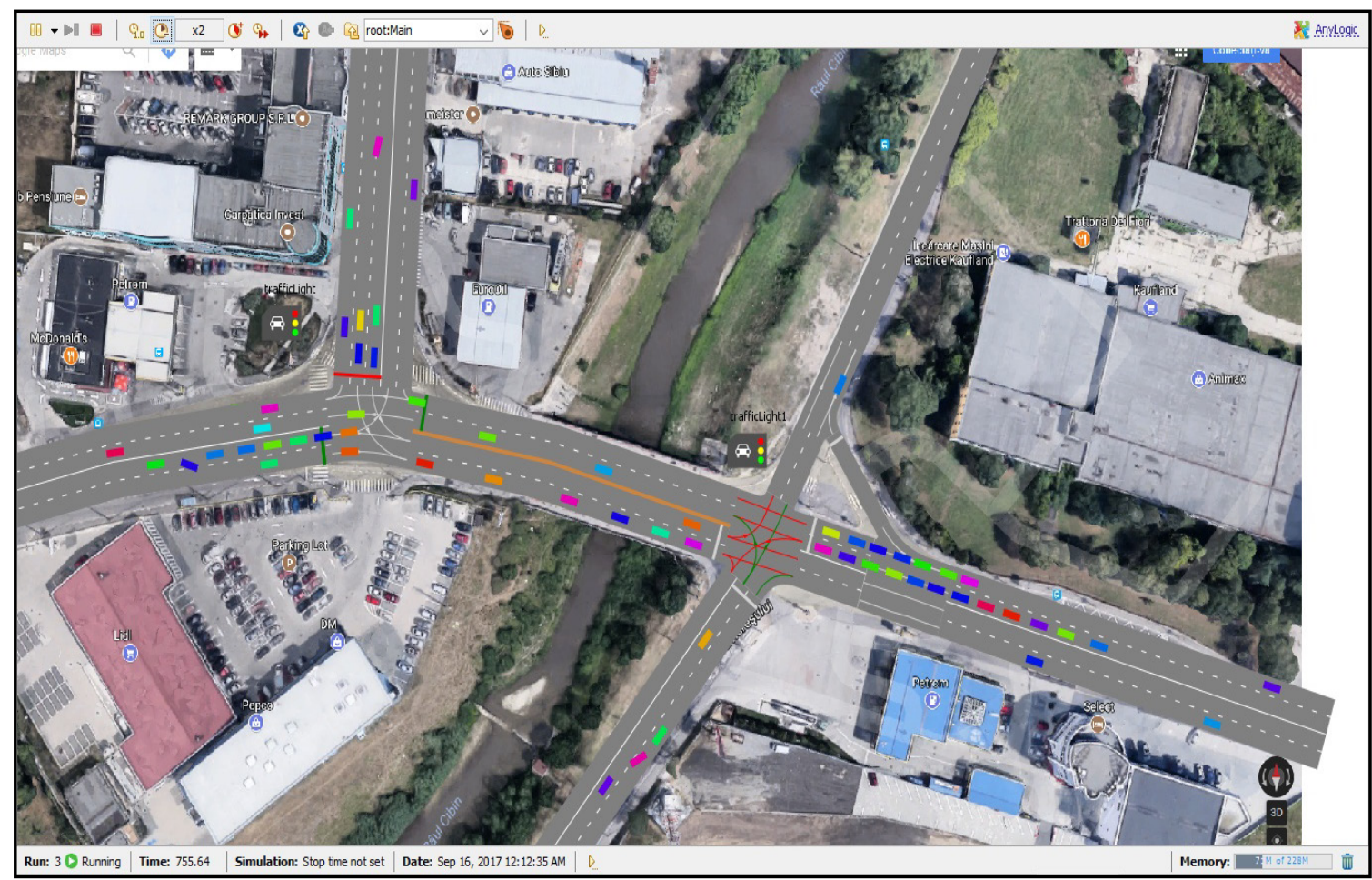

Figure no. 4 The road traffic simulation

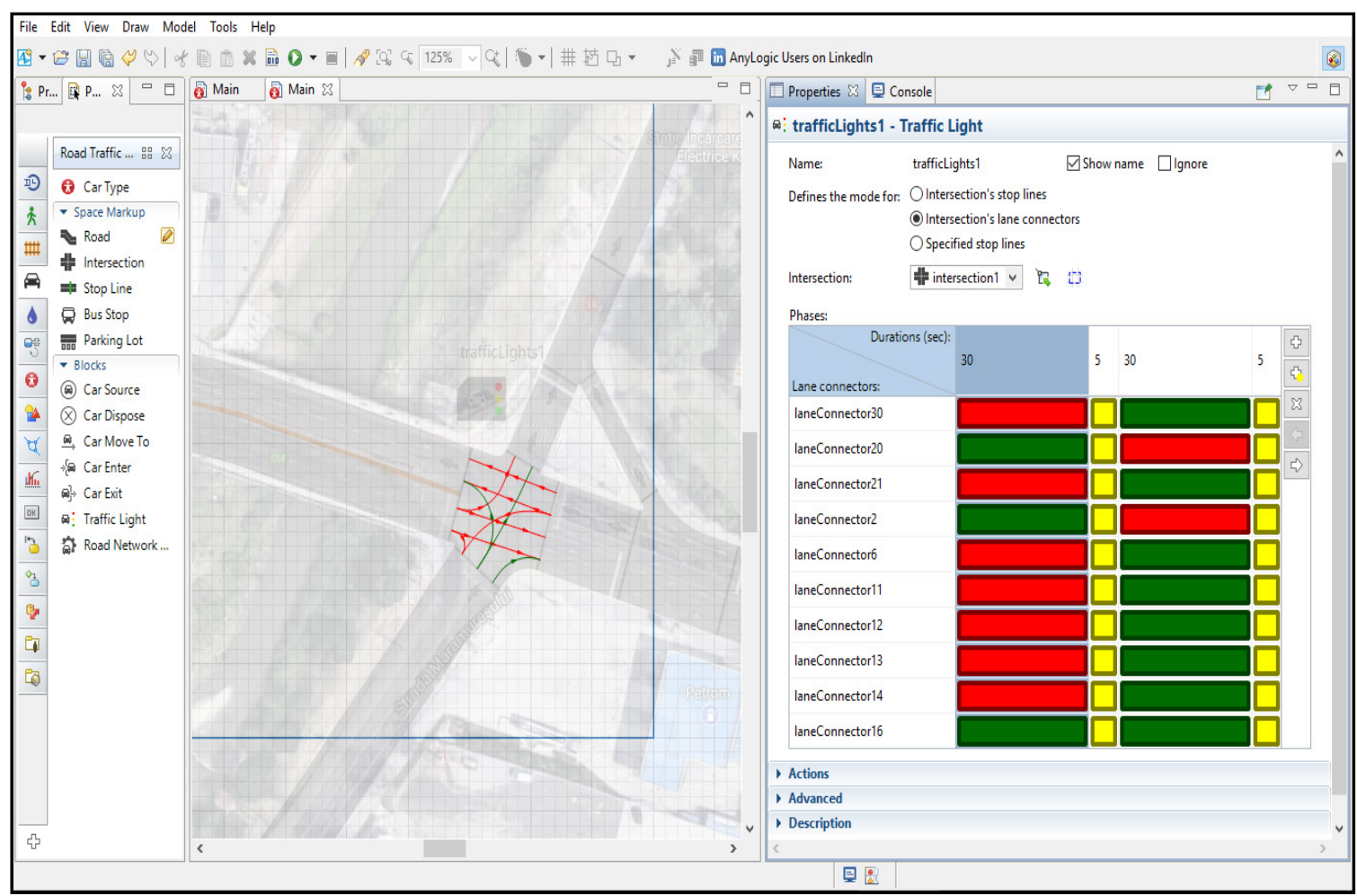

Figure no. 5 The traffic lights setup 
Step 4. Optimization of traffic lights

Firstly, it has been added a car type that knows the time when it enter the model. In the Figure no. 6 it is noticed that the average duration is 63.345 seconds for an simulation experiment running time about 10 minutes (600 seconds).

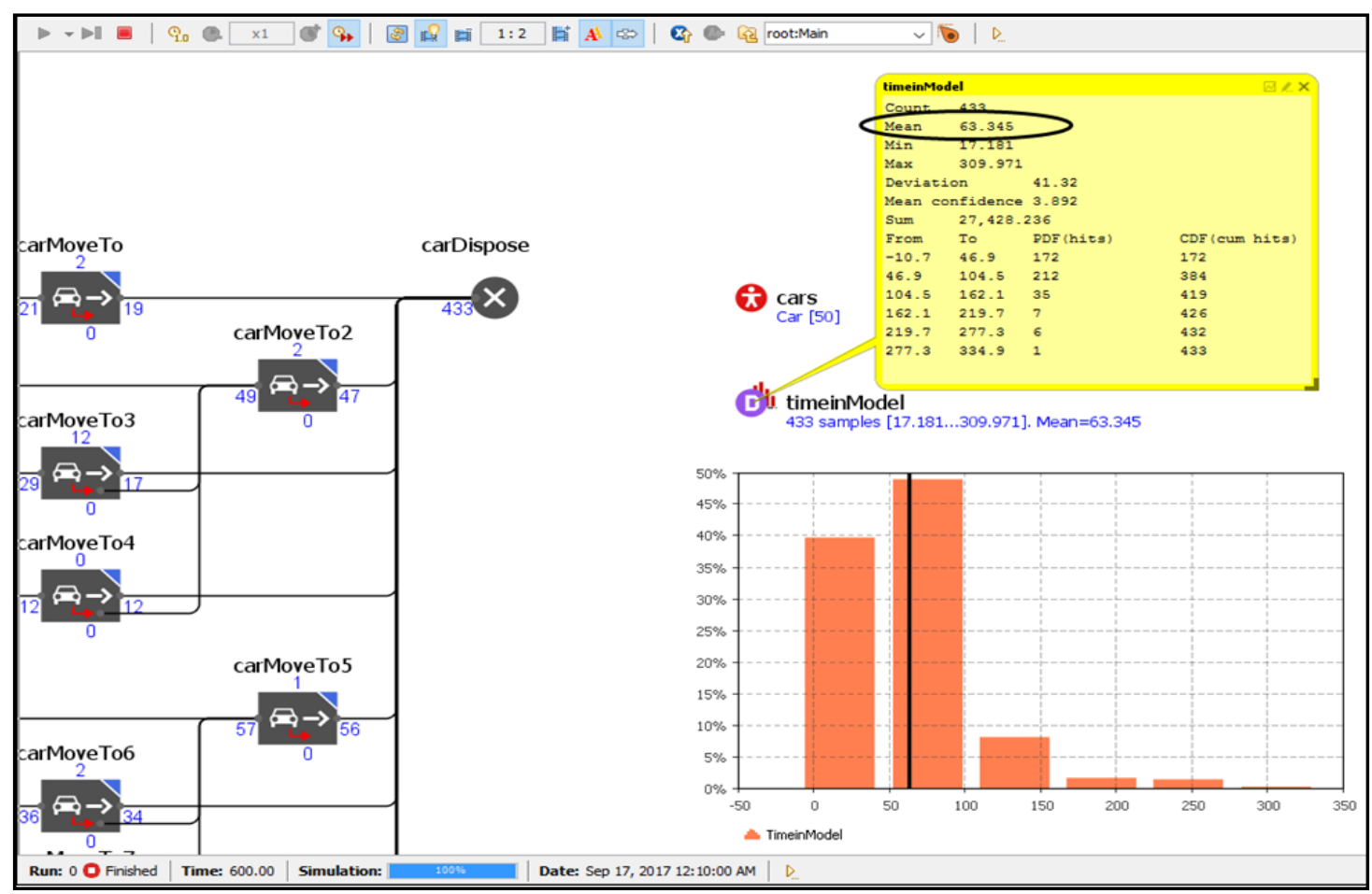

Figure no. 6 The simulation experiment

Next, the transportation has been optimized by reducing the traffic lights phases. Each phase duration was parameterized; therefore, it was used four parameters with the default value 30 seconds. Then, the duration of each phase of the traffic lights were changed in accordance with the established parameters: $\mathrm{p} 1$ and $\mathrm{p} 2$ for trafficLights; 33 and $\mathrm{p} 4$ for trafficLights1.

For optimization of urban transportation, it has been created and run a new optimization experiment. The purpose of the optimization experiment was to search for a parameter set corresponding to the best value of the provided objective function.

Optimization under uncertainty is supported by using replications. The AnyLogic displays the optimization progress chart during the iterations of simulation.

In order to optimize the experiment of the traffic flow all the parameters were changed from fixed values to discrete values ranging from 10 to 35 seconds with steps of 5 seconds. Then, for running the optimization experiment, the default UI has been created (in our case were run about 200 iterations).

In the Figure no. 7, the chart visually illustrates the optimization process. The $\mathrm{X}$-axis represents simulations and the Y-axis represents current objective, best infeasible objective, and best feasible objective found for each simulation. Next step is to copy the best feasible values for each parameter and paste them into the original simulation experiment. 


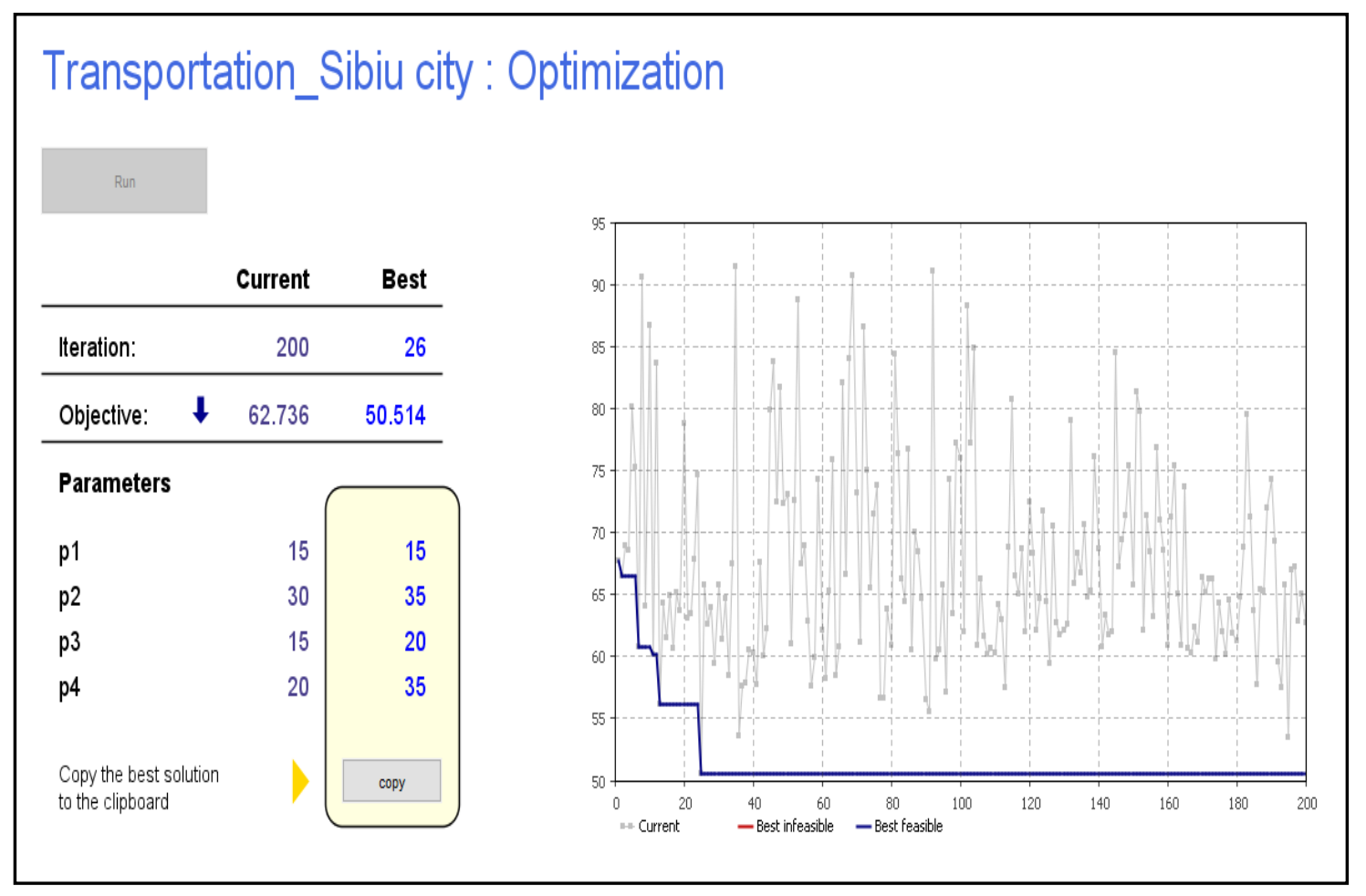

Figure no. 7 The optimization experiment

As it is seen in the chart, with the new $\quad \mathrm{p} 1=15 \mathrm{~s}, \mathrm{p} 2=35 \mathrm{~s}, \mathrm{p} 3=20 \mathrm{~s}$, and $\mathrm{p} 4=35 \mathrm{~s}$. values obtained from running of the Therefore, after optimization the mean optimization experiment related the traffic value decreased from 63.345 seconds to lights, the parameters were changed with 50.514 seconds (Figure no. 8).

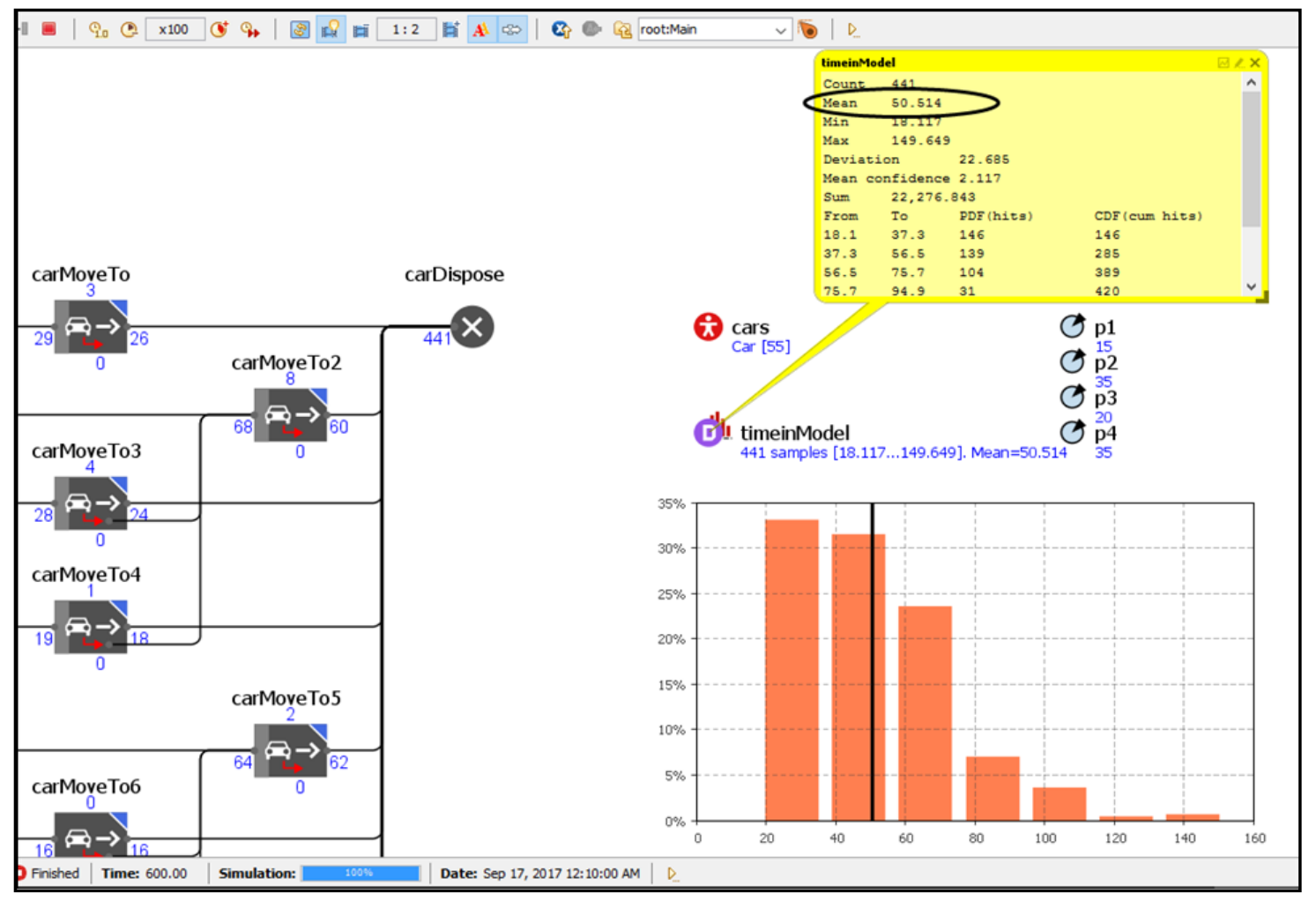

Figure no. 8 Running of the optimized simulation 


\section{Conclusions}

Nowadays, the optimization of vehicle traffic flow is an important fact, which appears in a crowded urban area, where the transportation system must function in a proper manner.

The AnyLogic software can also be used for adding more actors into the traffic flows. By focusing on the agent-based modeling, the urban transportation system can be simulated and analyzed after appropriate simulation iterations. The other actors belonging to urban transportation system could influence the simulation modeling and experimentation. The parking lots, bus stations, the vehicle types, and pedestrians simulated in the urban transportation model influence the optimization results.

"Agent-based modeling and simulation (ABMS) is a new approach to modeling systems comprised of autonomous, interacting agents. Applications range from modeling agent behavior in the stock market, supply chains, and consumer markets, to predicting the spread of epidemics, mitigating the threat of bio-warfare, and understanding the factors that may be responsible for the fall of ancient civilizations" (Macal \& North, 2009).

\section{REFERENCES}

Huynh, N., Cao, V. L., Wickramasuriya, R., Berryman, M., Perez, P., \& Barthelemy, J. (2015). Infrastructure and the City, An Agent Based Model for the Simulation of Transport Demand and Land Use, available at http://discovery.ucl.ac.uk/1469210/1/67-72.pdf.

Macal, C. M., \& North, M. J. (2009). Agent-based modeling and simulation, Proceedings of the 2009 Winter Simulation Conference, Texas, USA, 1.

Primăria Sibiu. (2017). Oraşul Sibiu, available at: http://www.sibiu.ro/index.php/ $\underline{\text { sibiu/prezentare. }}$

Puentes, R. (2015). The Avenue. Rethinking urban traffic congestion to put people first, available at https://www.brookings.edu/blog/the-avenue/2015/08/27/rethinking-urban-trafficcongestion-to-put-people-first/. 